\title{
NEW SHARP ERROR BOUNDS FOR SOME CORRECTED QUADRATURE FORMULAE
}

\author{
ZHENG LIU
}

\begin{abstract}
A generalization of the pre-Grüss inequality is used to derive a new sharp $L_{2}$ inequality which provides improved versions of some corrected inequalities that appear in the literature. An application to numerical integration is illustrated.
\end{abstract}

\section{INTRODUCTION}

In [4], the following sharp bounds for the errors in a unified corrected quadrature formulae are obtained:

Theorem 1. Let $f:[a, b] \rightarrow \mathbf{R}$ be such that $f^{\prime}$ is absolutely continuous on $[a, b]$ and $f^{\prime \prime} \in L_{2}[a, b]$, then for any $\theta \in[0,1]$,

$$
\begin{aligned}
& \mid \int_{a}^{b} f(t) d t-(b-a)\left[(1-\theta) f\left(\frac{a+b}{2}\right)+\theta \frac{f(a)+f(b)}{2}\right] \\
& -\frac{1-3 \theta}{24}(b-a)^{2}\left[f^{\prime}(b)-f^{\prime}(a)\right] \mid \leq \frac{(b-a)^{\frac{5}{2}}}{24 \sqrt{5}}\left(15 \theta^{2}-15 \theta+4\right)^{\frac{1}{2}} \sqrt{\sigma\left(f^{\prime \prime}\right)},
\end{aligned}
$$

where $\sigma(f)=\|f\|_{2}^{2}-\frac{1}{b-a}\left(\int_{a}^{b} f(t) d t\right)^{2}$ and $\|f\|_{2}:=\left[\int_{a}^{b} f^{2}(t) d t\right]^{\frac{1}{2}}$.

Inequality (1) is sharp in the sense that the constant $\frac{1}{24 \sqrt{5}}$ cannot be replaced by a smaller one.

Specifically, if we take $\theta=1,0, \frac{1}{3}, \frac{7}{15}$ and $\frac{1}{2}$ in (1), we obtain, respectively, the sharp corrected trapezoid type inequality, the sharp corrected midpoint type inequality, the sharp Simpson type inequality, the sharp corrected Simpson type inequality and the sharp corrected average midpointtrapezoid type inequality. These are:

2010 Mathematics Subject Classification. 26D15.

Key words and phrases. Sharp error bounds, corrected trapezoid type inequality, corrected midpoint type inequality, Simpson type inequality, corrected Simpson type inequality, corrected averaged midpoint-trapezoid type inequality. 


$$
\begin{gathered}
\left|\int_{a}^{b} f(t) d t-\frac{b-a}{2}[f(a)+f(b)]+\frac{(b-a)^{2}}{12}\left[f^{\prime}(b)-f^{\prime}(a)\right]\right| \leq \frac{(b-a)^{\frac{5}{2}}}{12 \sqrt{5}} \sqrt{\sigma\left(f^{\prime \prime}\right)} \\
\left|\int_{a}^{b} f(t) d t-(b-a) f\left(\frac{a+b}{2}\right)-\frac{(b-a)^{2}}{24}\left[f^{\prime}(b)-f^{\prime}(a)\right]\right| \leq \frac{(b-a)^{\frac{5}{2}}}{12 \sqrt{5}} \sqrt{\sigma\left(f^{\prime \prime}\right)} \\
\left|\int_{a}^{b} f(t) d t-\frac{b-a}{6}\left[f(a)+4 f\left(\frac{a+b}{2}\right)+f(b)\right]\right| \leq \frac{(b-a)^{\frac{5}{2}}}{12 \sqrt{30}} \sqrt{\sigma\left(f^{\prime \prime}\right)}, \\
\left|\int_{a}^{b} f(t) d t-\frac{b-a}{30}\left[7 f(a)+16 f\left(\frac{a+b}{2}\right)+7 f(b)\right]+\frac{(b-a)^{2}}{60}\left[f^{\prime}(b)-f^{\prime}(a)\right]\right| \\
\leq \frac{(b-a)^{\frac{5}{2}}}{60 \sqrt{3}} \sqrt{\sigma\left(f^{\prime \prime}\right)}
\end{gathered}
$$

and

$$
\begin{aligned}
\mid \int_{a}^{b} f(t) d t-\frac{b-a}{4}\left[f(a)+2 f\left(\frac{a+b}{2}\right)+f(b)\right]+ & \frac{(b-a)^{2}}{48}\left[f^{\prime}(b)-f^{\prime}(a)\right] \mid \\
& \leq \frac{(b-a)^{\frac{5}{2}}}{48 \sqrt{5}} \sqrt{\sigma\left(f^{\prime \prime}\right)} .
\end{aligned}
$$

Inequalities (2) and (3) have been considered in [1] and [2], the inequality (4) has been considered in [3] without a proof of its sharpness, while the corrected Simpson rule has been considered in [7], [8] and [5].

In [6], there is the following a generalization of the pre-Grüss inequality.

Lemma. Let $f, g, \Psi \in L_{2}(a, b)$, then,

$$
S_{\Psi}(f, g)^{2} \leq S_{\Psi}(f, f) S_{\Psi}(g, g),
$$

where

$$
\begin{aligned}
S_{\Psi}(f, g)=\int_{a}^{b} f(t) g(t) d t-\frac{1}{b-a} \int_{a}^{b} f(t) d t \int_{a}^{b} g(t) d t \\
\quad-\frac{1}{\|\Psi\|_{2}^{2}} \int_{a}^{b} f(t) \Psi(t) d t \int_{a}^{b} g(t) \Psi(t) d t
\end{aligned}
$$

and $\Psi$ satisfies

$$
\int_{a}^{b} \Psi(t) d t=0,
$$


while, as usual, $\|\cdot\|_{2}$ is the norm in $L_{2}(a, b)$. i.e.,

$$
\|\Psi\|_{2}^{2}=\int_{a}^{b} \Psi^{2}(t) d t
$$

In this paper, we use this generalization of the pre-Grüss inequality to derive a new sharp $L_{2}$ inequality which provides better estimation of error. An application in numerical integration is also considered.

\section{MAin RESUlts}

Theorem 2. Let the assumptions of Theorem 1 hold, then for any $\theta \in[0,1]$,

$$
\begin{aligned}
& \mid \int_{a}^{b} f(t) d t-(b-a)\left[(1-\theta) f\left(\frac{a+b}{2}\right)+\theta \frac{f(a)+f(b)}{2}\right] \\
& -\frac{1-3 \theta}{24}(b-a)^{2}\left[f^{\prime}(b)-f^{\prime}(a)\right] \mid \leq \frac{(b-a)^{\frac{5}{2}}}{24 \sqrt{5}} \sqrt{4-15 \theta+15 \theta^{2}} M(f ; a, b),
\end{aligned}
$$

where

$$
M(f ; a, b)=\left\{\left\|f^{\prime \prime}\right\|_{2}^{2}-\frac{\left[f^{\prime}(b)-f^{\prime}(a)\right]^{2}}{b-a}-\frac{\left[2 f^{\prime}\left(\frac{a+b}{2}\right)-f^{\prime}(a)-f^{\prime}(b)\right]^{2}}{b-a}\right\}^{\frac{1}{2}} .
$$

The inequality (10) is sharp.

Proof. Let

$$
p(t)= \begin{cases}1, & t \in\left[a, \frac{a+b}{2}\right] \\ -1, & t \in\left(\frac{a+b}{2}, b\right]\end{cases}
$$

and

$$
\Psi(t)= \begin{cases}\frac{(t-a)^{2}}{2}-\frac{\theta(b-a)}{2}(t-a)-\frac{1-3 \theta}{24}(b-a)^{2}, & t \in\left[a, \frac{a+b}{2}\right], \\ \frac{(t-b)^{2}}{2}+\frac{\theta(b-a)}{2}(t-b)-\frac{1-3 \theta}{24}(b-a)^{2}, & t \in\left(\frac{a+b}{2}, b\right],\end{cases}
$$

where $\theta \in[0,1]$.

It is not difficult to verify that

$$
\begin{aligned}
\int_{a}^{b} p(t) d t & =0, \\
\int_{a}^{b} \Psi(t) d t & =0, \\
\int_{a}^{b} p(t) \Psi(t) d t & =0 .
\end{aligned}
$$


Also,

$$
\begin{gathered}
\|p\|_{2}^{2}=\int_{a}^{b} p^{2}(t) d t=b-a \\
\|\Psi\|_{2}^{2}=\int_{a}^{b} \Psi^{2}(t) d t=\frac{(b-a)^{5}}{2880}\left(4-15 \theta+15 \theta^{2}\right),
\end{gathered}
$$

and

$$
\int_{a}^{b} f^{\prime \prime}(t) p(t) d t=2 f^{\prime}\left(\frac{a+b}{2}\right)-f^{\prime}(a)-f^{\prime}(b) .
$$

Integrating by parts,

$$
\begin{aligned}
\int_{a}^{b} f^{\prime \prime}(t) \Psi(t) d t=\int_{a}^{b} f(t) d t-(b-a) & {\left[(1-\theta) f\left(\frac{a+b}{2}\right)+\theta \frac{f(a)+f(b)}{2}\right] } \\
& -\frac{1-3 \theta}{24}(b-a)^{2}\left[f^{\prime}(b)-f^{\prime}(a)\right] .
\end{aligned}
$$

From (12), (14), (17) and (8),

$$
\begin{aligned}
S_{\Psi}\left(f^{\prime \prime}, p\right)= & \int_{a}^{b} f^{\prime \prime}(t) p(t) d t \\
& -\frac{1}{b-a} \int_{a}^{b} f^{\prime \prime}(t) d t \int_{a}^{b} p(t) d t \\
& \quad \frac{1}{\|\Psi\|_{2}^{2}} \int_{a}^{b} f^{\prime \prime}(t) \Psi(t) d t \int_{a}^{b} p(t) \Psi(t) d t \\
= & 2 f^{\prime}\left(\frac{a+b}{2}\right)-f^{\prime}(a)-f^{\prime}(b) .
\end{aligned}
$$

From (12), (14) and (8),

$$
\begin{aligned}
S_{\Psi}(p, p) & =\|p\|_{2}^{2}-\frac{1}{b-a}\left(\int_{a}^{b} p(t) d t\right)^{2}-\frac{1}{\|\Psi\|_{2}^{2}}\left(\int_{a}^{b} p(t) \Psi(t) d t\right)^{2} \\
& =b-a .
\end{aligned}
$$

From (16), (18) and (8),

$$
\begin{aligned}
S_{\Psi}\left(f^{\prime \prime}, f^{\prime \prime}\right)= & \left\|f^{\prime \prime}\right\|_{2}^{2}-\frac{1}{b-a}\left(\int_{a}^{b} f^{\prime \prime}(t) d t\right)^{2}-\frac{1}{\|\Psi\|_{2}^{2}}\left(\int_{a}^{b} f^{\prime \prime}(t) \Psi(t) d t\right)^{2} \\
= & \left\|f^{\prime \prime}\right\|_{2}^{2}-\frac{\left[f^{\prime}(b)-f^{\prime}(a)\right]^{2}}{b-a}-\frac{2880}{\left(4-15 \theta+15 \theta^{2}\right)(b-a)^{5}} \\
& \times\left\{\int_{a}^{b} f(t) d t-(b-a)\left[(1-\theta) f\left(\frac{a+b}{2}\right)+\theta \frac{f(a)+f(b)}{2}\right]\right. \\
& \left.-\frac{1-3 \theta}{24}(b-a)^{2}\left[f^{\prime}(b)-f^{\prime}(a)\right]\right\}^{2} \cdot(21)
\end{aligned}
$$


Thus from (19)-(21) and (7) we can obtain

$$
\begin{array}{r}
\frac{\left[2 f^{\prime}\left(\frac{a+b}{2}\right)-f^{\prime}(a)-f^{\prime}(b)\right]^{2}}{b-a} \leq\left\|f^{\prime \prime}\right\|_{2}^{2}-\frac{\left[f^{\prime}(b)-f^{\prime}(a)\right]^{2}}{b-a} \\
-\frac{2880}{\left(4-15 \theta+15 \theta^{2}\right)(b-a)^{5}}\left\{\int_{a}^{b} f(t) d t-(b-a)\left[(1-\theta) f\left(\frac{a+b}{2}\right)+\theta \frac{f(a)+f(b)}{2}\right]\right. \\
\left.\quad-\frac{1-3 \theta}{24}(b-a)^{2}\left[f^{\prime}(b)-f^{\prime}(a)\right]\right\}^{2}, \quad(22)
\end{array}
$$

which is equivalent to

$$
\begin{gathered}
\frac{2880}{\left(4-15 \theta+15 \theta^{2}\right)(b-a)^{5}}\left\{\int_{a}^{b} f(t) d t-(b-a)\left[(1-\theta) f\left(\frac{a+b}{2}\right)+\theta \frac{f(a)+f(b)}{2}\right]\right. \\
\left.-\frac{1-3 \theta}{24}(b-a)^{2}\left[f^{\prime}(b)-f^{\prime}(a)\right]\right\}^{2} \\
\leq\left\|f^{\prime}\right\|_{2}^{2}-\frac{[f(b)-f(a)]^{2}}{b-a}-\frac{\left[2 f^{\prime}\left(\frac{a+b}{2}\right)-f^{\prime}(a)-f^{\prime}(b)\right]^{2}}{b-a} .
\end{gathered}
$$

Inequality (10) follows from (23).

In order to prove that the inequality (10) is sharp, for any $\theta \in[0,1]$, we define the function,

$$
f(t)= \begin{cases}\frac{1}{24} t^{4}-\frac{\theta}{12} t^{3}, & t \in\left[0, \frac{1}{2}\right], \\ \frac{1}{24}(t-1)^{4}+\frac{\theta}{12}(t-1)^{3}+\frac{1-3 \theta}{24}\left(t-\frac{1}{2}\right), & t \in\left(\frac{1}{2}, 1\right]\end{cases}
$$

from which it follows that

$$
f^{\prime}(t)= \begin{cases}\frac{1}{6} t^{3}-\frac{\theta}{4} t^{2}, & t \in\left[0, \frac{1}{2}\right], \\ \frac{1}{6}(t-1)^{3}+\frac{\theta}{4}(t-1)^{2}+\frac{1-3 \theta}{24}, & t \in\left(\frac{1}{2}, 1\right]\end{cases}
$$

and

$$
f^{\prime \prime}(t)= \begin{cases}\frac{1}{2} t^{2}-\frac{\theta}{2} t, & t \in\left[0, \frac{1}{2}\right] \\ \frac{1}{2}(t-1)^{2}+\frac{\theta}{2}(t-1), & t \in\left(\frac{1}{2}, 1\right] .\end{cases}
$$

The function given in (24) is absolutely continuous since it is a continuous piecewise polynomial function.

We now suppose that (10) holds with a constant $K>0$ as

$$
\begin{aligned}
& \mid \int_{a}^{b} f(t) d t-(b-a)\left[(1-\theta) f\left(\frac{a+b}{2}\right)+\theta \frac{f(a)+f(b)}{2}\right] \\
& -\frac{1-3 \theta}{24}(b-a)^{2}\left[f^{\prime}(b)-f^{\prime}(a)\right] \mid \leq K(b-a)^{\frac{5}{2}} \sqrt{4-15 \theta+15 \theta^{2}} M(f ; a, b),
\end{aligned}
$$

where $M(f ; a, b)$ is as defined in (11). 
Choosing $a=0, b=1$, and $f$ as defined in (24), we get

$$
\begin{gathered}
\int_{0}^{1} f(t) d t=\frac{11-35 \theta}{1920}, \\
f(0)=0, \quad f(1)=\frac{1-3 \theta}{48}, \quad f\left(\frac{1}{2}\right)=\frac{1-4 \theta}{384} \\
f^{\prime}(0)=0, \quad f^{\prime}(1)=\frac{1-3 \theta}{24}, \quad f^{\prime}\left(\frac{1}{2}\right)=\frac{1-3 \theta}{48} \\
\int_{0}^{1}\left(f^{\prime \prime}(t)\right)^{2} d t=\frac{3-15 \theta+20 \theta^{2}}{960}
\end{gathered}
$$

such that the LHS of (28) becomes $\frac{4-15 \theta+15 \theta^{2}}{2880}$, and the $R H S$ becomes $\frac{K\left(4-15 \theta+15 \theta^{2}\right)}{24 \sqrt{5}}$.

Thus from (27), we find that $K \geq \frac{1}{24 \sqrt{5}}$, proving that the constant $\frac{1}{24 \sqrt{5}}$ is the best possible in (10).

Remark 1. It is obvious that the error estimation in (10) is better than that in (1).

Remark 2. If we take $\theta=1$ and $\theta=0$ in (10), we obtain the following sharp, corrected trapezoid type and corrected midpoint type inequalities, respectively, as

$\left|\int_{a}^{b} f(t) d t-\frac{b-a}{2}[f(a)+f(b)]+\frac{(b-a)^{2}}{12}\left[f^{\prime}(b)-f^{\prime}(a)\right]\right| \leq \frac{(b-a)^{\frac{5}{2}}}{12 \sqrt{5}} M(f ; a, b)$

and

$\left|\int_{a}^{b} f(t) d t-(b-a) f\left(\frac{a+b}{2}\right)-\frac{(b-a)^{2}}{24}\left[f^{\prime}(b)-f^{\prime}(a)\right]\right| \leq \frac{(b-a)^{\frac{5}{2}}}{12 \sqrt{5}} M(f ; a, b)$.

Remark 3. If $\theta=\frac{1}{3}$ in (10), we obtain a sharp Simpson type inequality of the form

$$
\left|\int_{a}^{b} f(t) d t-\frac{b-a}{6}\left[f(a)+4 f\left(\frac{a+b}{2}\right)+f(b)\right]\right| \leq \frac{(b-a)^{\frac{5}{2}}}{12 \sqrt{30}} M(f ; a, b),
$$

and if $\theta=\frac{7}{15}$ in (10), we obtain a sharp corrected Simpson type inequality of the form

$$
\begin{aligned}
\mid \int_{a}^{b} f(t) d t-\frac{b-a}{30}[ & {\left[7 f(a)+16 f\left(\frac{a+b}{2}\right)+7 f(b)\right] } \\
+ & \frac{(b-a)^{2}}{60}\left[f^{\prime}(b)-f^{\prime}(a)\right] \mid \leq \frac{(b-a)^{\frac{5}{2}}}{60 \sqrt{3}} M(f ; a, b) .
\end{aligned}
$$


From (30) and (31), we see that the corrected Simpson rule provides better results than the Simpson rule.

Remark 4. If $\theta=\frac{1}{2}$ in (10), we obtain the following sharp, corrected average midpoint-trapezoid type inequality,

$$
\begin{aligned}
\mid \int_{a}^{b} f(t) d t-\frac{b-a}{4}[ & \left.f(a)+2 f\left(\frac{a+b}{2}\right)+f(b)\right] \\
& +\frac{(b-a)^{2}}{48}\left[f^{\prime}(b)-f^{\prime}(a)\right] \mid \leq \frac{(b-a)^{\frac{5}{2}}}{48 \sqrt{5}} M(f ; a, b) .
\end{aligned}
$$

It is interesting to note that the smallest bound for (10) is obtained at $\theta=\frac{1}{2}$. Thus the corrected averaged midpoint-trapezoid rule is optimal in the current situation.

Remark 5. It is also clear that the error estimates in (28)-(32) are better than those in the corresponding results, (2)-(6).

\section{Applications in NUmerical integration}

Application here is to the averaged midpoint-trapezoid quadrature rule. Similar analysis can be performed on the other results considered in the previous section.

Theorem 3. Let $\pi=\left\{x_{0}=a<x_{1}<\cdots<x_{n}=b\right\}$ be a given subdivision of the interval $[a, b]$ such that $h_{i}=x_{i+1}-x_{i}=h=\frac{b-a}{n}$ and let the assumptions of Theorem 1 hold, then,

$$
\begin{aligned}
& \mid \int_{a}^{b} f(t) d t-\frac{h}{4} \sum_{i=0}^{n-1}\left[f\left(x_{i}\right)+2 f\left(\frac{x_{i}+x_{i+1}}{2}\right)+f\left(x_{i+1}\right)\right] \\
& \quad+\frac{(b-a)^{2}}{48 n^{2}}\left[f^{\prime}(b)-f^{\prime}(a)\right] \mid \leq \frac{(b-a)^{\frac{5}{2}}}{48 \sqrt{5} n^{2}} M_{n}(f) \leq \frac{(b-a)^{\frac{5}{2}}}{48 \sqrt{5} n^{2}} \sqrt{\sigma\left(f^{\prime \prime}\right)}
\end{aligned}
$$

where

$$
\begin{aligned}
M_{n}(f)=\left\{\left\|f^{\prime \prime}\right\|_{2}^{2}-\frac{\left[f^{\prime}(b)-f^{\prime}(a)\right]^{2}}{b-a}-\frac{1}{b-a}\left[f^{\prime}\left(x_{0}\right)+f^{\prime}\left(x_{n}\right)\right.\right. \\
\left.\left.+2 \sum_{i=1}^{n-1} f^{\prime}\left(x_{i}\right)-2 \sum_{i=0}^{n-1} f^{\prime}\left(\frac{x_{i}+x_{i+1}}{2}\right)\right]^{2}\right\}^{\frac{1}{2}} .
\end{aligned}
$$


Proof. From (32) in Remark 4,

$$
\begin{gathered}
\left|\int_{x_{i}}^{x_{i+1}} f(t) d t-\frac{h}{4}\left[f\left(x_{i}\right)+2 f\left(\frac{x_{i}+x_{i+1}}{2}\right)+f\left(x_{i+1}\right)\right]+\frac{h^{2}}{48}\left[f^{\prime}\left(x_{i+1}\right)-f^{\prime}\left(x_{i}\right)\right]\right| \\
\leq \frac{h^{\frac{5}{2}}}{48 \sqrt{5}}\left\{\int_{x_{i}}^{x_{i+i}}\left(f^{\prime \prime}(t)\right)^{2} d t-\frac{1}{h}\left[f^{\prime}\left(x_{i+1}\right)-f^{\prime}\left(x_{i}\right)\right]^{2}\right. \\
\left.-\frac{1}{h}\left[f^{\prime}\left(x_{i}\right)-2 f^{\prime}\left(\frac{x_{i}+x_{i+1}}{2}\right)+f^{\prime}\left(x_{i+1}\right)\right]^{2}\right\}^{\frac{1}{2}}
\end{gathered}
$$

By summing (35) over $i$ from 0 to $n-1$ and using the generalized triangle inequality, we obtain,

$$
\begin{gathered}
\left|\int_{a}^{b} f(t) d t-\frac{h}{4} \sum_{i=0}^{n-1}\left[f\left(x_{i}\right)+2 f\left(\frac{x_{i}+x_{i+1}}{2}\right)+f\left(x_{i+1}\right)\right]+\frac{h^{2}}{48}\left[f^{\prime}\left(x_{i+1}\right)-f^{\prime}\left(x_{i}\right)\right]\right| \\
\leq \frac{h^{\frac{5}{2}}}{48 \sqrt{5}} \sum_{i=0}^{n-1}\left\{\int_{x_{i}}^{x_{i+i}}\left(f^{\prime \prime}(t)\right)^{2} d t-\frac{1}{h}\left[f^{\prime}\left(x_{i+1}\right)-f^{\prime}\left(x_{i}\right)\right]^{2}\right. \\
\left.-\frac{1}{h}\left[f^{\prime}\left(x_{i}\right)-2 f^{\prime}\left(\frac{x_{i}+x_{i+1}}{2}\right)+f^{\prime}\left(x_{i+1}\right)\right]^{2}\right\}^{\frac{1}{2}}
\end{gathered}
$$

By using the Cauchy inequality twice, it can be seen that,

$$
\begin{gathered}
\sum_{i=0}^{n-1}\left\{\int_{x_{i}}^{x_{i+1}}\left(f^{\prime \prime}(t)\right)^{2} d t-\frac{1}{h}\left[f^{\prime}\left(x_{i+1}\right)-f^{\prime}\left(x_{i}\right)\right]^{2}\right. \\
-\frac{1}{h}\left[f^{\prime}\left(x_{i}\right)-2 f^{\prime}\left(\frac{x_{i}+x_{i+1}}{2}+f^{\prime}\left(x_{i+1}\right)\right]^{2}\right\}^{\frac{1}{2}} \\
\leq \sqrt{n}\left\{\left\|f^{\prime \prime}\right\|_{2}^{2}-\frac{n}{b-a} \sum_{i=0}^{n-1}\left[f^{\prime}\left(x_{i+1}\right)-f^{\prime}\left(x_{i}\right)\right]^{2}\right. \\
\left.-\frac{n}{b-a} \sum_{i=0}^{n-1}\left[f^{\prime}\left(x_{i}\right)-2 f^{\prime}\left(\frac{x_{i}+x_{i+1}}{2}\right)+f^{\prime}\left(x_{i+1}\right)\right]^{2}\right\}^{\frac{1}{2}} \\
\leq \sqrt{n}\left\{\left\|f^{\prime \prime}\right\|_{2}^{2}-\frac{\left[f^{\prime}(b)-f^{\prime}(a)\right]^{2}}{b-a}-\frac{1}{b-a}\left[f^{\prime}\left(x_{0}\right)+f^{\prime}\left(x_{n}\right)\right.\right. \\
\left.\left.+2 \sum_{i=1}^{n-1} f^{\prime}\left(x_{i}\right)-2 \sum_{i=0}^{n-1} f^{\prime}\left(\frac{x_{i}+x_{i+1}}{2}\right)\right]^{2}\right\}^{\frac{1}{2}}
\end{gathered}
$$

Consequently, the inequality (33) with (34) follow from (36) and (37). 
Acknowledgement. The author wishes to thank the referee for his helpful suggestions which have improved the presentation of this paper.

\section{REFERENCES}

[1] N. B. Barnett, P. Cerone and S. S. Dragomir, A sharp bound for the error in the corrected trapezoid rule and applications, Tamkang J. Math., 33 (2002), 253-258.

[2] P. Cerone, On perturbed trapezoidal and midpoint rules, Korean J. Comput. Appl. Math., 2 (2002), 423-435.

[3] S. S. Dragomir, Better bounds in some Ostrowski-Grüss type inequalities, RGMIA Research Report Collection 3, Article 3, 2000.

[4] Z. Liu, A sharp $L_{2}$ inequality of Ostrowski type, ANZIAM J., 49 (2008), 423-429.

[5] J. Pečarić and I. Franjić, Generalization of corrected Simpson's formula, ANZIAM J., 47 (2006), 367-385.

[6] N. Ujević, A generalization of the pre-Grüss inequality and applications to some qradrature formulae, J. Inequal. Pure Appl. Math., 3 (2) (2002), Art. 13.

[7] N. Ujević, A generalization of the modified Simpson's rule and error bounds, ANZIAM J., 47 (E) (2005), E1-E13.

[8] N. Ujević and A. J. Roberts, A corrected quadrature formula and applications, ANZIAM J., 45 (E) (2004), E41-E56.

(Received: July 26, 2011)

(Revised: January 16, 2012)
Institute of Applied Mathematics

School of Science

University of Science and Technology Liaoning Anshan 114051, Liaoning

China

E-mail: lewzheng@163.net 